\title{
ЭХОКАРДИОГРАФИЧЕСКАЯ ОЦЕНКА МОРФОЛОГИЧЕСКИХ ОСОБЕННОСТЕЙ СТРОЕНИЯ СЕРДЦА У ДЕТЕЙ ГРУДНОГО ВОЗРАСТА
}

Эхокардиография позволяет проводить неинвазивную оценку размеров и функций сердца и является важным инструментом для оценки состояния сердца у детей и взрослых [1]. Поскольку размеры камер сердца могут изменяться с ростом соматических клеток в детском возрасте, важно установить эхокардиографические измерения в соответствии с размером тела [2]. Существует большое количество опубликованных в нашей и зарубежной литературе данных эхокардиографических исследований сердца у детей $[2,3]$. При этом они являются противоречивыми и не учитывают анатомические особенности сердечно-сосудистой системы в ранние периоды постнатального онтогенеза. Кроме того, на стандарты роста населения могут влиять экологические, социальные и экономические факторы этого региона, в нашем случаи Донецкого [4].

Это требует разработки региональных эхокардиографических справок. Чтобы интерпретировать количественные эхокардиографические данные, размеры сердца должны сравниваться с нормальными значениями. Эталонный диапазон значений каждого сердечного измерения должен оцениваться из популяции нормальных сердец.

На сегодняшний день доступно более точное измерение размеров сердца, поскольку эхокардиографическая технология развивается [5]. Следовательно, биологические и технологические изменения позволяют обновить актуальные эталонные значения для сердечных измерений здоровых новорожденных и детей.

\section{ЦЕЛ В РАБОТЫ}

Установить количественные показатели линейных параметров сердца и магистральных сосудов в области его основания у детей грудного возраста Донецкого региона.

\section{МАТЕРИАЛ И МЕТОДЫ}

Исследования проведены на 80 детях в возрасте от одного месяца до года, у которых от- сутствовали признаки заболеваний сердечнососудистой системы и другой соматической патологии. Они были поделены на две группы по половому признаку. Первую группу составили- 42 ребенка мужского пола, вторую группу - 38 женского. Методом ультразвукового исследования сердца в М- и В-режимах измеряли и оценивали линейные показатели сердца, такие как толщина межжелудочковой перегородки (ТМЖП) в систолу и диастолу, конечный диастолический размер левого желудочка (КДРлж), конечный систолический размер левого желудочка (КСРлж), толщину задней стенки левого желудочка (ЗСЛЖ) в систолу и диастолу, конечный диастолический размер правого желудочка (КДРпж), диаметр аорты (АО).

Все количественные параметры обрабатывали статистическими методами. Данные представлены в тексте в виде среднеарифметической и ошибки средней и медианы с минимальными и максимальными значениями, а так же вносились в таблицы в виде $\mathrm{Me}(\mathrm{Q} 1-\mathrm{Q} 3)$, где Ме - медиана, Q1 - первый квартиль и Q3 - третий квартиль. Полученные результаты проверены на нормальность (обе группы), закон распределения отличается от нормального на уровне значимости $\mathrm{p}<0,05$. Проведено сравнение центральных тенденций двух независимых выборок по критерию Манна-Уитни. В ходе исследования был проведен корреляционный анализ Спирмена.

\section{РЕЗУЛЬТАТЫ И ОБСУЖДЕНИЕ}

Анализ антропометрических показателей детей грудного возраста (см. табл.) показал, что средние значения массы тела и ошибка средней у мальчиков составляли 6,0 0,33 кг, при медиане 6,1 кг. Минимальные и максимальные показатели колебались в пределах 3,4-9,5 кг. Сред-

(c) В.А. Васильев, К.А. Мурейси, Р.В. Басий,

И.В. Коктышев, П.А. Лепихов, 2020

(C) Университетская Клиника, 2020 
Линейные показатели структур сердца в группах наблюдения по данным УзИ Ме(Q1-Q2)

Таблица.

\begin{tabular}{lcc}
\hline \multirow{2}{*}{ Показатели } & \multicolumn{2}{c}{ Дети грудного возраста 1-12 мес., $\mathrm{n}=80$} \\
\cline { 2 - 3 } & $\begin{array}{c}\text { мальчики } \mathrm{n}=42 \\
\text { 1группа }\end{array}$ & $\begin{array}{c}\text { девочки } \mathrm{n}=38 \\
\text { 2группа }\end{array}$ \\
\hline \hline Вес, кг & $6,1(3,9-8,0)$ & $5,0(3,9-6,5)$ \\
\hline Рост, см & $65,0(60,0-71,0)$ & $60,0(58,0-65,0)$ \\
\hline Площадь тела, ${ }^{2}$ & $0,28(0,23-0,37)$ & $0,27(0,23-0,33)$ \\
\hline КДРпж & $0,85(0,81-0,93)$ & $0,89(0,81-0,93)$ \\
\hline ТМЖПд, см & $0,48(0,43-0,5)$ & $0,47(0,4-0,5)$ \\
\hline КДРлж, см & $2,23(1,94-2,61)$ & $2,22(1,88-2,56)$ \\
\hline ЗСЛЖд, см & $0,48(0,42-0,51)$ & $0,48(0,44-0,5)$ \\
\hline ТМЖПс, см & $0,65(0,57-0,7)$ & $0,61(0,57-0,67)$ \\
\hline КСРлж, см & $1,35(1,25-1,55)$ & $1,3(1,25-1,55)$ \\
\hline ЗСЛЖс, см & $0,63(0,57-0,72)$ & $0,62(0,57-0,67)$ \\
\hline АО, см. & $1,28(1,15-1,42)$ & $1,19(1,08-1,3)$ \\
\hline
\end{tabular}

ние значения массы тела у девочек достигали

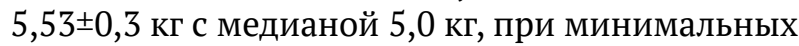
параметрах 3,2 кг и максимальных 9,3 кг.

Средние значения по ростовому показателю у мальчиков выше, чем у девочек 65,45ะ1,26 cм, медиана составляла 65,0 см, при колебании роста от минимального 49,0 см до максимального 81,0 см. У девочек средние значения роста достигали 61,92 $\pm 1,15$ см, а медиана 60,0 см, при их минимальном росте 51,0 см и максимальном 85,0 cM.

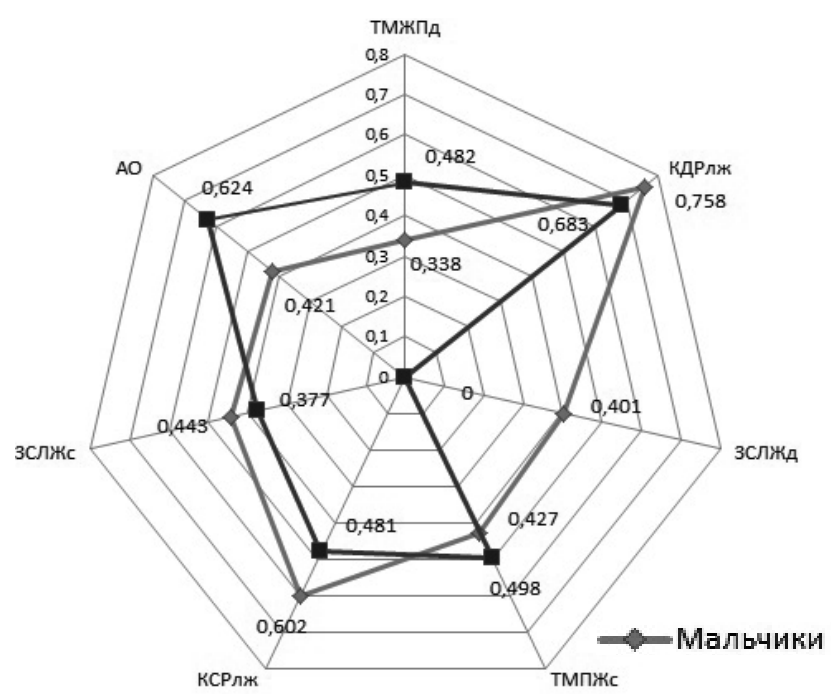

Рис. 1. Корреляционная связь линейных показателей сердца с весом в группе мальчиков и группе девочек.
Площадь тела у лиц мужского пола достигала $0,30 \pm 0,08 \mathrm{M} 2$, а $\mathrm{Me}=0,28$ м $^{2}$ при минимальных значениях 0,18 м2 и максимальных 0,55 м². У лиц женского пола средние значения площа-

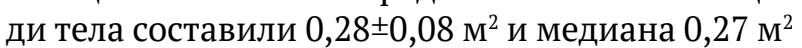
с колебанием параметров площади тела между 0,14-0,57 $\mathrm{M}^{2}$.

Были проанализированы линейные показатели по данным ультразвукового сканирования средние значения толщины межжелудочковой перегородки в диастолу у мальчиков 0,47士0,008

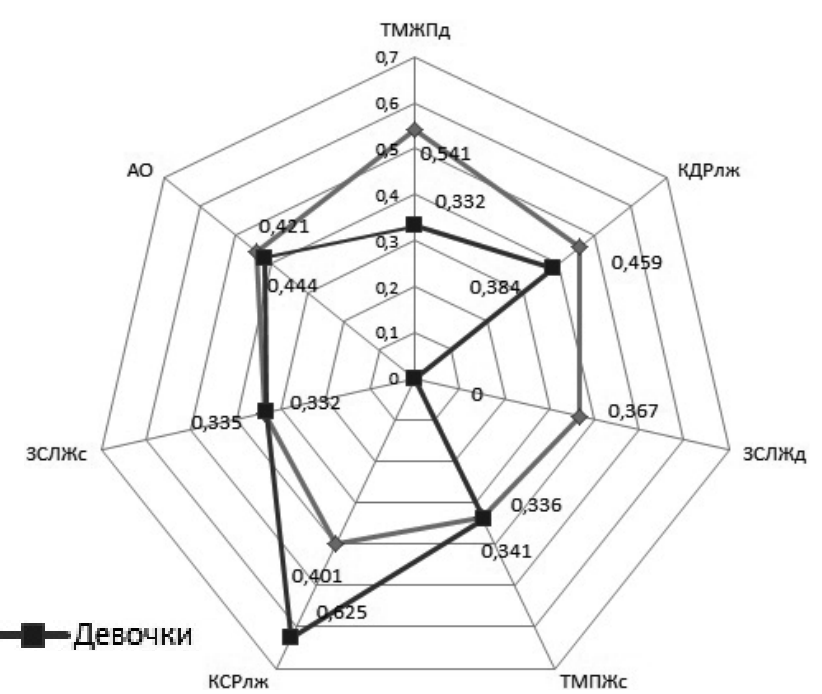

Рис. 2. Корреляционная связь линейных показателей сердца с ростом в группе мальчиков и группе девочек. 


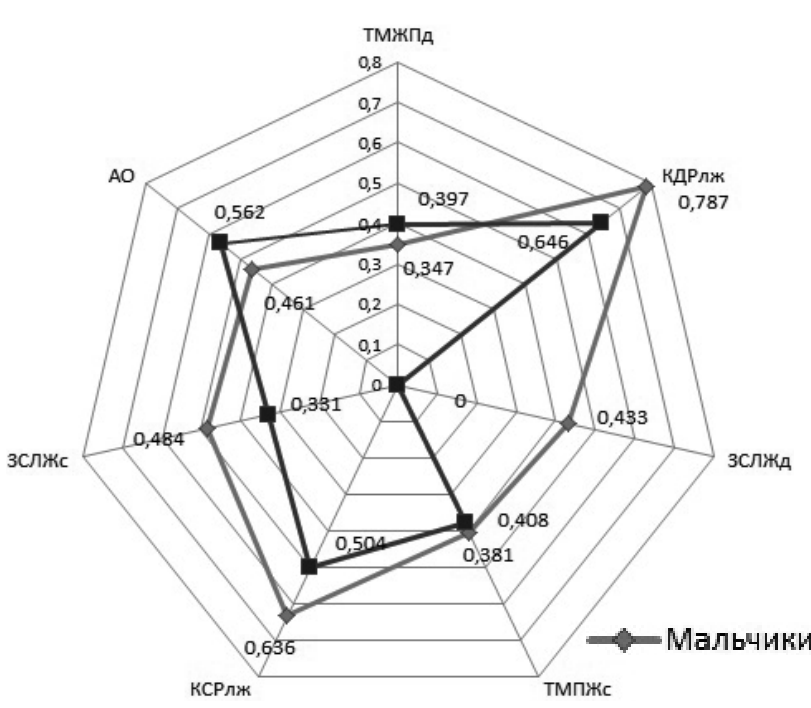

Рис. 3. Корреляционная связь линейных показателей сердца с возрастом в группе мальчиков и группе девочек.

см и с медианой 0,48 см, при минимальном значении $-0,35$ см и максимальном - 0,58 см. Средние значения толщины межжелудочковой перегородки в период диастолы у девочек $0,45 \pm 0,01$ см, медиана 0,47 cм, с колебаниями параметров толщины межжелудочковой перегородки от 0,32 до 0,72 см.

Толщина межжелудочковой перегородки в систолу у мальчиков составила $0,63 \pm 0,01 \mathrm{~cm}$, а медианы 0,65 см., при размахе данных от 0,37 до 0,82 см. У девочек средние значения того же показателя совпадали с медианой и достигали 0,61 $\pm 0,01$ см. Минимальные показатели межжелудочковой перегородки фиксировали на 0,33 см, а максимальные на 0,83 см.

Средние значения толщины задней стенки левого желудочка в период диастолы у мальчиков и девочек составляли $0,47 \pm 0,01 \mathrm{~cm}$, медиана 0,48 см в обеих группах. Разница в интервале колебаний толщины стенки левого желудочка у мальчиков составляла от 0,3 до $0,66 \mathrm{~cm}$, а у девочек от 0,3 до 0,72 см.

Толщина задней стенки левого желудочка в систолу у мальчиков $-0,65 \pm 0,01 \mathrm{~cm}$, при $\mathrm{Me}=0,63$ см и при минимальных показателях толщины задней стенки 0,52 см и максимальных 0,92 см. Средние значения и медиана задней стенки левого желудочка у девочек совпадали и достигали $0,62 \pm 0,01$ см. Минимальные и максимальные параметры колебались в интервале от 0,37 до 0,85 CM.

Конечный диастолический размер левого желудочка у детей первой группы равен $2,27 \pm 0,05$ cм, $\mathrm{Me}=2,23$ см от 1,62 до 2,97 см. Во второй группе средние значения конечного диастолическо-

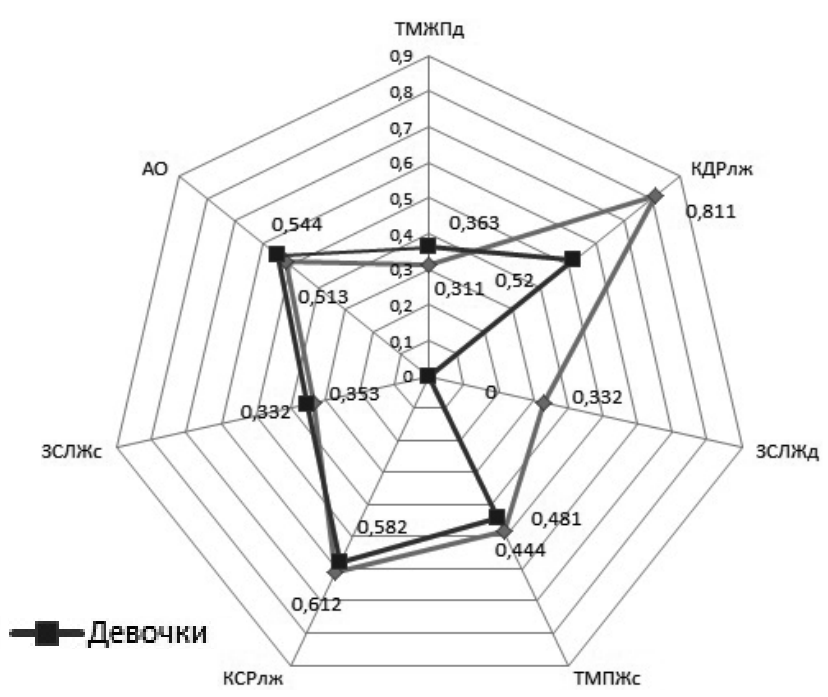

Рис. 4. Корреляционная связь линейных показателей сердца с площадью тела в группе мальчиков и группе девочек.

го размера левого желудочка составляли всего $2,24 \pm 0,06$ см, а медиана 2,22 см. Минимальный параметр конечного диастолического размера начинался с уровня 1,57 см, а максимальный доходил до 3,0 см.

Конечный систолический размер левого желудочка у мальчиков $1,4 \pm 0,03$ см при медиане 1,35 см и интервале колебаний от 1,03 до 1,86 см. У девочек средние значения гораздо ниже и достигали $1,32 \pm 0,03$ см с медианой 1,3 см, при этом минимальные и максимальные параметры находились в интервале от 1,03 до 1,87 см.

Средние значения диаметра аорты у мальчиков $-1,30 \pm 0,03$ см, медиана 1,28 см., при этом минимальные показатели диаметра аорты составили 1,0 см, а максимальные - 2,0 см. У девочек средние значения достигали $1,21 \pm 0,03$ см с медианой 1,19 см, при минимальном параметре 0,94 см в то время как максимальный показатель совпадал с параметром у мальчиков $-2,0$ см.

В ходе исследования выявлена положительная корреляционная связь средней силы в обеих группах детей грудного возраста между весом, ростом, возрастом и площадью тела детей с толщиной межжелудочковой перегородки в систолу и диастолу, конечным диастолическим размером левого желудочка, конечным систолическим размером левого желудочка, диаметром аорты (рис. 1. - рис. 4.). Обращает на себя внимание отсутствие корреляционной связи у девочек между толщиной задней стенки левого желудочка в диастолу с весом, ростом, возрастом и площадью тела. 
Проведено сравнение центральных тенденций двух независимых выборок для всех показателей: вес, рост, площадь тела детей, толщина межжелудочковой перегородки в систолу и диастолу, конечный диастолический размер левого желудочка левого желудочка, конечный систолический размер левого желудочка, толщину задней стенки левого желудочка в систолу и диастолу, конечный диастолический размер правого желудочка, конечный диастолический и конечный систолический объемы левого желудочка, исследовали диаметр аортального отверстия, по критерию Манна-Уитни. Выявлены статистически значимые отличия диаметра аорты между мальчиками и девочками на уровне значимости $\mathrm{p}=0,038$. У мальчиков этот показатель выше, чем у девочек, по остальным показателям все различия, которые имеются между группами, не являются статистически значимыми р>0,05.

\section{В Ы В 0 д Ы}

Таким образом, в ходе исследования были определены линейные количественные показатели сердца мальчиков и девочек грудного возраста. Выявлены статистически значимые отличия диаметра аорты между ними. Проведен корреляционный анализ исследуемых показателей, установлена корреляционная связь средней силы между линейными показателями и антропометрическими данными.

\section{В.А. Васильев, К.А. Мурейси, Р.В. Басий, И.В. Коктышев, П.А. Лепихов.}

ГОО ВПО «Донецкий национальный медицинский университет имени М. Горького», Донецк

\section{ЭХОКАРДИОГРАФИЧЕСКАЯ ОЦЕНКА МОРФОЛОГИЧЕСКИХ ОСОБЕННОСТЕЙ СТРОЕНИЯ СЕРДЦА У ДЕТЕЙ ГРУДНОГО ВОЗРАСТА}

Эхокардиография позволяет проводить неинвазивную оценку размеров и функций сердца и является важным инструментом для оценки состояния сердца у детей и взрослых. Несмотря на большое количество эхокардиографических исследований, опубликованных для детей различных регионов, в них до конца не раскрыты особенности роста и развития сердца и сосудов, отсутствуют данные получения количественных изменений в Донецком регионе. Целью данного исследования было установление количественных показателей линейных параметров сердца и магистральных сосудов в области его основания у детей грудного возраста Донецкого региона. Исследования проведены на 80 детях в возрасте от одного месяца до года, у которых отсутствовали признаки заболеваний сердечно-сосудистой системы. Они были поделены на две группы по половому признаку. Первую группу составили- 42 ребенка мужского пола, вторую группу - 38 женского пола. Методом ультразвукового исследования в М- и В-режимах измеряли и оценивали линейные показатели такие как толщина межжелудочковой перегородки, конечный диастолический размер левого желудочка, конечный систолический размер левого желудочка, конечный диастолический размер правого желудочка. толщину задней стенки левого желудочка, диаметр аорты. В ходе проделанной работы были определены линейные количественные показатели сердца мальчиков и девочек грудного возраста. Выявлены статистически значимые отличия диаметра аорты между ними. Проведен корреляционный анализ исследуемых показателей, установлена корреляционная связь средней силы.

Ключевые слова: ультразвуковое исследование сердца, аорта, легочный ствол, дети от одного месяца до года.

\section{V.A. Vasiliev, K.A. Mureisy, R.V. Basiy, I.V. Koktyshev, P.A. Lepihov}

SEI HPE «M. Gorky Donetsk National Medical University», Donetsk

\section{ECHOCARDIOGRAPHIC ASSESSMENT OF MORPHOLOGICAL CHARACTERISTICS OF HEART STRUCTURE IN CHILDREN OF INFANTS}

Echocardiography allows a non-invasive assessment of the size and function of the heart and is an important tool for assessing the state of the heart in children and adults. Despite the large number of echocardiographic studies published for children of different regions, they did not fully reveal the features of growth and development of the heart and blood vessels, and there is no data on quantitative changes in the Donetsk region. The purpose of this study was to establish quantitative indicators of the linear parameters of the heart and major vessels in the area of its base in infants of the Donetsk region. The studies conducted 80 children aged from one month to a year who did not have signs of diseases of the cardiovascular system. They were divided into two groups by gender. The first group consisted of 42 male children, the second group - 38 female. Linear indicators such as interventricular septum thickness, final diastolic size of the left ventricle, final systolic size of the left ventricle, final diastolic size of the right ventricle, the thickness of the posterior wall of the left ventricle, the diameter of the aorta were measured and evaluated using ultrasound in $\mathrm{M}$ - and B-modes. In the course of the work, linear quan- 
titative indicators of the heart of boys and girls of infancy were determined. Statistically significant differences were revealed in the diameter of the aorta between them. A correlation analysis of the studied indicators was car- ried out; a correlation relationship of medium strength was established.

Key words: ultrasound examination of the heart, aorta, pulmonary trunk, children from one month to a year.

\section{ЛИТЕРАТУРА}

1. Cavarretta E., Maffessanti F., Sperandii F., Guerra E., Ouaranta F., et al. Reference values of left heart echocardiographic dimensions and mass in male peri-pubertal athletes. European Journal of Preventive Cardiology. 2018; 25(11): 1204-1215.

2. Гелашвили О.А., Хисамов Р.Р., Шальнева И.Р. Физическое развитие детей и подростков. Современные проблемы науки и образования. 2018; 3. URL: https://science-education.ru/pdf/2018/3/27656.pdf

3. Тарасова А.А, Филюшкина М.Н, Ефимов М.С., Чабаидзе Ж.Л. Ультразвуковая оценка морфофункционального состояния сердца у недоношенных детей в течение первого года жизни. Вестник Российского научного центра рентгенорадиологии Минздрава России. 2013; $3(13): 6$.

4. Буракова Е.Н. Динамика изменений антропометрических показателей у детей Самарского региона в постнатальном периоде онтогенеза : автореф. дис. ... канд. мед. наук Оренбург; 2016. 21.

5. Классен А.А. Ультразвуковая анатомия сердца человека на этапах онтогенеза: корреляция анатомических срезов с двухмерными ультрозвуковыми изображениями. Ультразвуковая и функциональная диагностика. 2015; $4: 86$.

\section{REFERENCE}

1. Cavarretta E., Maffessanti F., Sperandii F., Guerra E., Quaranta F., et al. Reference values of left heart echocardiographic dimensions and mass in male peri-pubertal athletes. European Journal of Preventive Cardiology. 2018; 25(11): 1204-1215.

2. Gelashvili O. A., Hisamov R. R., Shal'neva I. R. Fizicheskoe razvitie detej i podrostkov [Physical development of children and adolescents]. Sovremennye problemy nauki i obrazovaniya. 2018; 3. URL: https://science-education.ru/ pdf/2018/3/27656.pdf (in Russian).

3. Tarasova A.A, Filyushkina M.N, Efimov M.S., Chabaidze Zh.L. Ultrazvukovaya otsenka morfofunktsionalnogo sostoyaniya serdtsa $\mathrm{u}$ nedonoshennyih detey $\mathrm{v}$ techenie pervogo goda zhizni [Ultrasonic assessment of morphofunctional heart condition in premature babies during first year of life]. Vestnik Rossiyskogo nauchnogo tsentra rentgenoradiologii Minzdrava Rossii. 2013; 3 (13): 6 (in Russian).

4. Burakova, E. N. Dinamika izmeneniy antropometricheskih pokazateley u detey Samarskogo regiona v postnatalnom periode ontogeneza [Dynamics of changes in anthropometric indicators in children of the Samara region in the postnatal period of ontogenesis]: avtoref. dis. ... kand. med. Nauk. Orenburg; 2016. 21 (in Russian).

5. Klassen A.A. Ultrazvukovaya anatomiya serdtsa cheloveka na etapah ontogeneza: korrelyatsiya anatomicheskih srezov s dvuhmernyimi ultrozvukovyimi izobrazheniyami [Ultrasound anatomy of the human heart at the stages of ontogenesis: correlation of anatomical sections with twodimensional ultrasound images]. Ultrazvukovaya i funktsionalnaya diagnostika. 2015; 4: 86 (in Russian). 